\title{
Diseño de una plataforma virtual de autoaprendizaje de la escritura académica: fundamentación teórica y decisiones pedagógicas en la Universidad de Chile'
}

Design of a Virtual Platform for Self Instructional of Academic Writing: Theoretical Fundamentals and Pedagogical Decisions at the University of Chile

\author{
PAULA GONZÁLEZ-ÁLVAREZ \\ Universidad de Chile \\ Chile \\ p.gonzalezalvarez@u.uchile.cl
}

(Recibido: I5-O7-20I6; aceptado: IO-I2-2OI6)

Resumen. Desde 2014, la Universidad de Chile ha venido desarrollando un laboratorio virtual de escritura académica para apoyar el desarrollo de las habilidades de producción de textos de todos sus estudiantes. Este artículo presenta el modelo de diseño de esta plataforma a partir de antecedentes teóricos acerca de la didáctica de la escritura y la enseñanza de la escritura en entornos virtuales. Para ello, detalla los contenidos y la estructura de los materiales ofrecidos y su articulación en versiones de acceso libre y de orientación disciplinar. Finalmente, se reflexiona acerca del papel de un laboratorio virtual de escritura en el aprendizaje de las literacidades académicas y las responsabilidades institucionales para fomentar su desarrollo.

Palabras clave: escritura; redacción; enseñanza superior; herramienta de autoformación; aprendizaje en línea.
Abstract. Since 2014, the University of Chile has been developing an online writing lab to support the development of writing skills in all of its students. This article presents the design model for this platform, according to the literature on the teaching of writing and online writing instruction. The article details the structure and contents offered in the platform and its organization into general access and discipline-oriented versions. Finally, the article reflects on the role of an online writing lab in achieving academic literacies and the institutional responsibilities associated with the development of writing skills.

Keywords: writing; composition; higher education; self-instructional aids; electronic learning.

\footnotetext{
${ }^{\text {I }}$ Para citar este artículo: González Álvarez, Paula (20I8). Diseño de una plataforma virtual de autoaprendizaje de la escritura académica: fundamentación teórica y decisiones pedagógicas en la Universidad de Chile. Alabe 1\%. [www.revistaalabe.com]

DOI: IO.I5645/Alabe20I8.I7.7
} 


\section{Contexto sobre las necesidades de instrucción en escritura en las universidades chilenas}

Tradicionalmente, en Chile ha existido la creencia de que las escuelas son las responsables de desarrollar las competencias de lectura y escritura de los estudiantes. Existe una alta preocupación por el percibido "mal desempeño" de los jóvenes que ingresan a la educación superior, ya que al momento de su ingreso no han desarrollado adecuadamente las habilidades que necesitan para trabajar con textos escritos ni mucho menos para producirlos a conformidad de los docentes universitarios. Sin embargo, en los últimos quince años se ha popularizado la noción de que estas habilidades requieren ser aprendidas y desarrolladas en el contexto mismo de la formación universitaria, ya que escribir y comunicarse académicamente requiere el manejo no solo de los aspectos mecánicos de la lectura y la escritura, sino que también de las convenciones disciplinares de cada comunidad (Carlino, 20I3). Es decir, la escuela no puede "preparar" a los estudiantes porque la cultura universitaria difiere de la escolar en muchos aspectos críticos, como la forma en que el estudiante debe comprobar sus aprendizajes. Por ejemplo, la escuela normalmente requiere que el estudiante repita información, mientras que la universidad exige no solo que el estudiante pueda dar cuenta de ideas ajenas, identificando adecuadamente a sus autores, sino que también pueda generar sus propios planteamientos y defenderlos ante la evidencia teórica y empírica (Carlino, 2003).

El abordaje de la instrucción en escritura se ha dado de dos grandes formas en Chile desde hace unos diez años. En primer lugar, un número cada vez mayor de carreras, en ámbitos variados como la ingeniería, las ciencias sociales o los oficios técnicos, asigna un espacio curricular en primer año para un curso regular de habilidades comunicativas, lo que responde al modelo de formación general que es frecuente en universidades anglosajonas (cf. Hillard \& Harris, 2003). En el contexto local, estos cursos suelen concebirse desde una perspectiva de remediación o nivelación, y se centran en aspectos de corrección ortográfica o gramatical, aunque también suele incluirse la lectura de textos académicos sencillos y la escritura de textos académicos transversales, como un ensayo argumentativo. En algunos casos, se ha adoptado una perspectiva más ecológica: se diseñan cursos específicos para diferentes carreras o áreas de estudio, para así abordar la lectura y escritura desde una perspectiva más disciplinar (cf. Acevedo et al., 2OI3; Ávila, González-Álvarez \& Peñaloza, 2OI3; González-Álvarez, 2OI5a). En segundo lugar, algunas carreras incluyen el desarrollo de las habilidades comunicativas de manera transversal en los cursos disciplinares, lo que se identifica con la tradición anglosajona de la escritura a través del currículum. Ejemplo de ello son propuestas didácticas en cursos de pedagogía de ciclo básico o fonoaudiología (Concha \& Miño, 20I3; Peñaloza, 20I3). Con todo, la enseñanza de la escritura en la educación superior chilena suele toparse con restricciones, como una elevada carga académica que relega los cursos de escritura al último lugar de importancia, o una resistencia por parte de los docentes a incorporar actividades que implican más tiempo de planificación y de revisión (cf. González-Álvarez, 20I4). En este 
contexto, formular nuevas iniciativas de desarrollo de la escritura requiere identificar estas restricciones y ofrecer acciones que puedan ser adoptadas fácilmente por la comunidad universitaria.

En el caso de la Universidad de Chile, no hubo sino hasta 20I4 una propuesta institucional de desarrollo de la escritura; las iniciativas existentes solo se desarrollaban de manera local en algunos cursos de carreras específicas (cf. Peñaloza, 20I3). El Departamento de Pregrado, dependiente de la Vicerrectoría de Asuntos Académicos, formuló en 2013 un proyecto para instalar institucionalmente el desarrollo de las habilidades comunicativas escritas mediante la conformación de un programa con dos grandes líneas de trabajo: la asesoría a docentes para incluir la escritura en las materias, y el desarrollo de materiales de autoaprendizaje para la escritura. Se planteó que estos contenidos se encontraran a libre disposición de la comunidad universitaria para servir como un apoyo contingente a las necesidades transversales de los estudiantes de la institución. Durante 2OI4, estas dos líneas de trabajo se institucionalizaron en el Programa de Lectura y Escritura Académica (LEA UChile), inscrito en la Unidad de Aprendizaje del Departamento de Pregrado².

El objetivo de este artículo es detallar el proceso de diseño, desarrollo e implementación de la plataforma de autoaprendizaje de la escritura del Programa LEA UChile, denominada "LEA lab" 3 . En primer lugar, se exponen algunos antecedentes teóricos para informar las decisiones de diseño del material didáctico. Luego, se indica el proceso de diseño de los cursos y la plataforma en que se alojan. A continuación se reportan algunas dificultades sobre el proceso de puesta en marcha y su relación con nuevas decisiones estratégicas para la continuidad de la iniciativa. Finalmente, en las conclusiones se discute la relación de este proyecto con las prácticas de instrucción efectiva de la escritura.

\section{Antecedentes teóricos relevantes para el diseño de materiales}

En primer lugar, para la construcción de la plataforma se escogió un enfoque didáctico. El Programa LEA defiende un aprendizaje situado de la escritura, basado en decisiones consensuadas por la comunidad, por lo que existía desde el principio un interés por alejarse de materiales excesivamente prescriptivos. Así, se optó por iniciar el trabajo de diseño con una sistematización de buenas prácticas en instrucción en escritura, tanto en aula presencial como en entornos virtuales. Como parte de esa indagación, en primer lugar, se reconocieron y analizaron tres grandes enfoques metodológicos para la enseñanza de la escritura que debían considerarse en el diseño:

\footnotetext{
${ }^{2}$ Más información sobre el programa se encuentra en el sitio web http://miaprendizaje.uchile.cl.

${ }^{3}$ La plataforma se encuentra disponible en el sitio web http://laboratoriolea.uchile.cl. Esta es de libre acceso previa inscripción con número de identidad.
} 
I) El modelo de producto, particularmente el enfoque gramatical (Cassany, I99O y Parodi, I999), que se centra en el dominio mecánico de los aspectos gramaticales de la lengua para producir un buen texto. Este modelo no tiene mayor vigencia en los estudios contemporáneos sobre composición y se ha demostrado que el dominio gramatical no tiene mayor impacto en la calidad textual ni en la capacidad para corregir errores (Andrews et al., 2006 y Martínez Gómez, 2OII). Sin embargo, en el contexto universitario puede ofrecer algunas ventajas para proporcionar, desde la descripción gramatical, una serie de mecanismos para mejorar el estilo y la cohesión textuales, una vez que los elementos de contenido y estructura hayan sido desarrollados desde otro enfoque. Es decir, tiene una utilidad limitada, pero real, en aspectos de revisión textual (Williams et al., I985).

2) El modelo de proceso se centra en las acciones y estrategias cognitivas y metacognitivas que deben emprender los escritores para poder producir. Propio de este modelo es el enfoque procesual, en el que se privilegia la enseñanza de estrategias concretas para abordar el proceso de escritura y sus etapas recursivas: planificación, textualización, revisión y edición (cf. Didactext, 2003 para la propuesta del contexto hispanohablante). Este modelo es muy utilizado en la actualidad, pero suele combinarse con otros enfoques didácticos, ya que una atención exclusiva en los procesos no permite abordar aspectos contextuales importantes, es decir, los criterios que justifican la elección de una u otra estrategia, considerando que el proceso no ocurrirá siempre de la misma forma y dependerá de las características de los estudiantes, de los textos a producir y de los contextos de producción.

3) El modelo contextual reconoce que la producción dependerá de las características contextuales, de audiencia y de los textos mismos y que, por lo tanto, no puede desarrollarse una didáctica generalista. Propio de este modelo es el enfoque funcional, surgido en contextos de enseñanza de segunda lengua, y que busca enseñar una serie de tipos textuales que puedan adaptarse a situaciones comunicativas concretas (Cassany, I990). En la misma línea se ubica el enfoque de género, que busca detectar, analizar y caracterizar los textos que circulan en una comunidad discursiva particular, para así explicitar criterios que permitan a los integrantes de esa comunidad escribir los textos propios de ella (Swales, I990 y Bhatia, 2002). Este modelo también es muy utilizado en la actualidad, pero se debe ser cauteloso en su uso ya que es posible que la didáctica caiga en una prescripción (es decir, que se establezcan estructuras y contenidos rígidos, sin atender al contexto particular de cada acto de producción). En contextos universitarios, estos enfoques contextuales pueden combinarse con la enseñanza de enfoque procesual. En efecto, una de las últimas líneas pedagógicas ha desarrollado un enfoque de género $y$ proceso que combina la enseñanza de un género particular con la explicitación de estrategias para facilitar el proceso de producción (Badger \& White, 2000; Kim \& Kim, 2005; Nordin \& Mohammad, 2006 y González-Álvarez \& Izquierdo, 20I4).

Como se mencionó con anterioridad, las iniciativas más frecuentes de nivelación en escritura adoptan principalmente un enfoque funcional y gramatical, con énfasis en la corrección y el aprendizaje de modelos más bien rígidos. Sin embargo, tras el análisis de 
las recomendaciones teóricas, se decidió que el diseño de nuestra plataforma requeriría la combinación de estrategias de los tres tipos de modelos, como se reseñará en la sección siguiente.

\section{Consideraciones para el desarrollo de un curso virtual}

Respecto a las propuestas didácticas para enseñar escritura en entornos virtuales, las experiencias locales (cf. Ávila, González-Álvarez y Peñaloza, 2OI3 y Ávila y GonzálezÁlvarez, 20I5a, 20I6) han mostrado que un curso virtual puede seguir una estructura similar a los cursos presenciales en la medida en que las rutas de trabajo expliciten y modelen la escritura como proceso. Considerando que el modelo de la Web 2.o, dominante en la actualidad, privilegia una interacción con plataformas que permitan no solo el acceso a contenidos, sino que también su manipulación y creación (McManus, 2005 citado en Greenhow, Robelia y Hughes, 2009), en un curso virtual es importante propiciar la interacción con la plataforma, con el tutor y con otros estudiantes para promover la construcción colaborativa del conocimiento. Sin embargo, y considerando que para este proyecto se buscaba generar una plataforma de acceso libre, sin restricciones de tiempo asociadas, las posibilidades de interacción en tiempo real con otros estudiantes o tutores son muy limitadas. Por ello, se prefirió desarrollar un modelo de interacción automatizada con la plataforma a través de ejercicios con retroalimentación inmediata y, como complemento, pautas de autoevaluación para uso privado del estudiante. Este modelo, aunque tiene desventajas en lo interpersonal, permite una mayor flexibilidad y más oportunidades para desarrollar cognitivamente las habilidades trabajadas (Mick \& Middlebrook, 2005).

Respecto a las decisiones metodológicas, a partir de los antecedentes revisados se propuso combinar aspectos didácticos de los modelos contextuales y de proceso, principalmente, apoyados con estrategias del enfoque gramatical para resolver problemas puntuales de escritura. Así, los materiales producidos incorporan, en primer lugar, la ejercitación de la escritura como un proceso en el que se puede trabajar con pasos, no necesariamente sucesivos: planificación de un género discursivo específico, textualización con atención a las convenciones del lenguaje académico, y revisión a partir de aspectos normativos del español. Así, se definieron algunos ejes temáticos básicos para la generación de materiales:

- Estrategias generales de lectura para extraer, analizar y sintetizar información de textos académicos.

- Estructura general y secuencias típicas de textos académicos argumentativos y expositivos.

- Convenciones estilísticas del lenguaje académico, incluyendo aspectos como la precisión conceptual o el uso de estrategias de impersonalización.

- Aspectos normativos del español, incluyendo ortografía y normas de cohesión textual. 
- Intertextualidad en textos académicos y convenciones de citación.

En el diseño, también se consideró que los materiales generados debían cumplir con requisitos mínimos de accesibilidad (Martínez \& Olsen, 2OI5 y Warnock, 2OI5). La interacción con una plataforma de desarrollo de la escritura requiere, necesariamente, que el usuario tenga buenas habilidades de lectura, ya que el medio natural para comprender la escritura es precisamente el texto escrito. Así, se espera del estudiante que posea una competencia retórica (Sánchez, González \& García, 2002) adecuadamente desarrollada para comprender los contenidos. Sin embargo, un buen diseño didáctico procurará eliminar la mayor cantidad de obstáculos lingüísticos para acceder y hacer uso de los recursos disponibles. Así, los materiales didácticos debieron cumplir con las siguientes características:

- Estar escritos en lenguaje común, sin uso de tecnicismos o definiciones de conceptos lingüísticos, excepto cuando estos sean estrictamente necesarios para el desarrollo de una habilidad, en cuyo caso deben definirse y explicarse en términos sencillos.

- Apelar directamente al estudiante, utilizando la segunda persona singular.

- Incorporar ejemplos de problemas frecuentes, para los cuales se debe ofrecer una explicación y al menos una propuesta de solución.

- Incluir plantillas de planificación o pautas de cotejo descargables para poder ejercitar las habilidades trabajadas en textos reales.

\section{Diseño de los cursos}

El proyecto inicial consideró preliminarmente una diferenciación de materiales de acuerdo con dos criterios: especificidad de los contenidos (generales o contextualizados a un área disciplinar) y posibilidades de retroalimentación de ejercicios (automatizada, entre pares y con un tutor). Por razones prácticas, en el diseño se abandonó la retroalimentación entre pares, ya que el acceso libre a los contenidos no permitiría calendarizar esta clase de revisión de manera sistemática. En el caso de la retroalimentación por tutor, se consideró que esta solo podía asociarse a un programa estructurado de desarrollo de los contenidos; la retroalimentación automatizada se asoció al acceso libre a los módulos.

Así, el diseño final de los cursos propuso, por una parte, dos secciones de acceso libre a los contenidos agrupados en cursos: una para la comunidad en general y otra contextualizada a las carreras de ingeniería. Por otra parte, propuso la creación de un curso general que incorporara todos los contenidos y estrategias generados y los trabajara ordenadamente.

\section{Cursos de acceso libre}

Para el diseño de estos cursos se contrató a una diseñadora instruccional que colaboró con el equipo en la construcción de rutas específicas de trabajo según las áreas temáticas de los materiales y las necesidades básicas de los estudiantes. Este trabajo derivó 
en un curso de lectura y tres de escritura, como se señala a continuación:

I. Estrategias de lectura: curso que aborda de manera general la habilidad de comprensión lectora. Entrega materiales para desarrollar las capacidades de selección, análisis y síntesis de la información académica y la generación de productos de lectura.

2. Planificación de textos: curso que explicita la estructura y secuencias textuales típicas de los textos argumentativos y expositivos. Incluye lineamientos para construir una introducción y una conclusión efectivas de acuerdo con sus movidas retóricas frecuentes.

3. Estilo académico: curso que ofrece criterios para adecuar el lenguaje a las convenciones académicas referidas al registro, la variedad léxica, la impersonalización, el uso de párrafos y los mecanismos de citación.

4. Ortografía y cohesión: curso que ejercita las normas ortográficas literales, acentuales y puntuales del español, así como la sintaxis oracional y la conexión entre oraciones.

Como se mencionó, para estos materiales se generaron algunas versiones contextualizadas a las carreras de ingeniería. La adaptación consistió principalmente en la incorporación de ejemplos y convenciones propias de las áreas científicas (por ejemplo, la utilidad de las definiciones de conceptos matemáticos en lenguaje común) y la creación de materiales nuevos para responder a necesidades específicas (como el uso de tablas y gráficos en informes).

\section{Curso estructurado general}

De acuerdo con algunas experiencias previas de cursos virtuales de escritura en el entorno local (cf. Ávila et al., 2013 y Ávila y González-Álvarez, 2OI4, 2OI6), se diseñó un gran curso que incorporaba todos los contenidos en función del desarrollo de un proyecto de escritura. Las propuestas de escritura por proyectos (Stoller, 2002) tienen la ventaja de permitir ejercitar las habilidades en un contexto real y asociado a un producto final susceptible de ser evaluado por otros; la asociación de este proyecto a una temática relevante para la disciplina en la que se inscribe la carrera de un estudiante también permite que este se familiarice con dicha temática u otros tópicos de interés académico, lo que, en el caso de ingresantes a la universidad, se configura como una oportunidad muy rica de inserción a la vida universitaria. Así, los contenidos se dispusieron en una lógica de progresiva incorporación de habilidades a medida que se avanzaba en el proyecto:

- En primer lugar, se ejercita la búsqueda de fuentes académicas y la lectura comprensiva de estas, para realizar un primer acercamiento al tópico investigado.

- En segundo lugar, se presenta la estructura de los géneros a producir (un ensayo argumentativo o un informe expositivo) y estrategias generales de planificación.

- En tercer lugar, se inicia la textualización del género mediante entregas sucesivas de escritura. Para cada entrega se consulta con anterioridad material referido a aspectos de norma del español, de estilo académico y de convenciones de citación. 
- En cuarto lugar, se ejercita la escritura de la introducción y la conclusión mediante la revisión de su estructura básica y una nueva planificación a partir del desarrollo ya generado.

- Finalmente, se consolida el trabajo en un texto unitario y se revisaron aspectos de presentación formal para un contexto académico.

El curso contempla una duración oficial de siete semanas con una entrega de avance semanal y estaba dirigido a estudiantes de primer año. Se puede apreciar que el desarrollo de este curso requiere un acompañamiento para el estudiante pues, aunque la instrucción virtual busca la autonomía del estudiante, las personas necesitan el apoyo de un mentor para desarrollar la capacidad de trabajar autónomamente (cf. Feldman, Konold \& Coulter, 2000, citado en Warschauer, 2007). Además, el desarrollo de las habilidades de escritura requerirá un monitoreo constante del proceso y un reforzamiento de las estrategias cuando esto sea necesario. En el caso de este curso se incorporó un equipo de tutores pares de escritura, quienes fueron previamente formados en aspectos teóricos y didácticos de la instrucción en escritura en entornos virtuales. Cada tutor trabajaba con un grupo pequeño de hasta 5 estudiantes, todos pertenecientes a su misma área disciplinar o carreras afines, con el propósito de compartir con el grupo su experiencia como miembro de esa comunidad y su familiaridad con los tópicos abordados.

La formulación de este curso resultó muy novedosa por varios motivos. En primer lugar, la decisión de incorporar tutores pares responde a la potencialidad de este sistema para acercar a los estudiantes de primer año al mundo académico de una manera más amable, acompañado por otro estudiante que ha vivido muy recientemente esta misma experiencia de incorporación (Alzate-Medina \& Peña Borrero, 2OIO) y que, por tanto, propicia un trabajo con textos de poco riesgo evaluativo y más permisivos ante las equivocaciones (low stakes writing, en palabras de Warnock, 20I5). En segundo lugar, la perspectiva de un miembro pleno de cada comunidad disciplinar permite relacionar de manera más clara los contenidos de escritura con la producción de un texto inserto en las preocupaciones de la carrera de cada estudiante, lo que le otorga mayor autenticidad al ejercicio. En tercer lugar, la forma de trabajo permite a los tutores tomar un rol protagonista en la generación de contenidos que permitan contextualizar aún más el trabajo o contestar adecuadamente a las necesidades emergentes de su grupo (Warnock, 20I5). Finalmente, esta forma de trabajo valida la perspectiva y experiencia de un tutor que, si bien no es experto aún ni en su área ni en temas de escritura, es un interlocutor valioso para el enriquecimiento de la experiencia de aprender a escribir en la universidad.

\section{Denominación de la plataforma}

Hasta este punto, y con el diseño terminado, solo bastaba dar a la plataforma un nombre atractivo para la comunidad. En concordancia con las tendencias de los países angloparlantes, particularmente de la tradición norteamericana, se propuso adoptar el concepto de writing lab. Así, la plataforma se nombró "Laboratorio de lectura y escritura académica" o "LEA lab". Sus áreas abiertas se denominaron "laboratorio abierto" para 
la versión general y "laboratorio de ingeniería” para la contextualizada, respectivamente; la versión estructurada en un curso se denominó "laboratorio dirigido". Además, para hacer un guiño a los online writing labs que incorporan la figura de una lechuza (en inglés owl, que se identifica con la sigla de estos laboratorios), en los materiales gráficos se incorporó la figura de un chuncho, ave rapaz nacional que es también uno de los símbolos tradicionalmente utilizados para representar a la Universidad de Chile y, en particular, a su club deportivo (ver ejemplo en sección siguiente).

\section{Montaje en plataforma}

El sistema gestor de contenidos (CMS, por su sigla en inglés) escogido para montar los cursos fue Moodle 2.8.3. Se escogió esta plataforma pues permitía el montaje de diversos tipos de recursos y, además, facilitaba el registro de los estudiantes para efectos de seguimiento y estadísticas de uso. Los contenidos, en su mayoría, fueron incorporados en productos de aprendizaje en formato HTML5 con el programa Articulate Storyline previamente al montaje en los cursos diseñados. Se cauteló, para el proceso de desarrollo de la plataforma, que esta fuera accesible en diferentes sistemas operativos, incluyendo, cuando fuera posible, aquellos usados en dispositivos móviles (principalmente iOS y Android).

Para el montaje también se contempló la definición de una identidad gráfica de la plataforma. En la práctica, esto consistió en la determinación de una paleta de colores basada en el logotipo del Programa LEA, la generación de gráficos de bienvenida a los cursos, y el uso estratégico de colores dentro de los contenidos para diferenciar temas y unidades.

\section{¡Bienvenido a}

\section{Estrategias de lectura!}

En este curso aprenderás algunos criterios para seleccionar, priorizar y ordenar la información de los textos que lees para la universidad. Además, aprenderás a aprovechar y optimizar tus procesos de lectura.

El curso tiene dos módulos de aprendizaje y el tiempo estimado para completar las actividades es de 6 y 10 horas cronológicas respectivamente.

Estudia cada módulo, autoevalúate y revisa los documentos complementarios que hemos puesto a tu disposición.

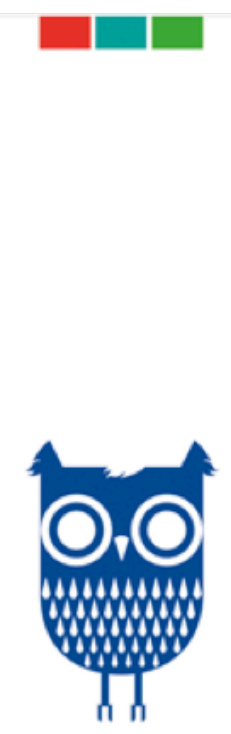

Figura 1. Ejemplo de bienvenida a curso, con propuesta gráfica 


\section{Implementación: aciertos, errores y desafíos}

Luego de un período de prueba de dos meses, se inauguró oficialmente la plataforma durante el segundo semestre de 2015 para uso de la comunidad en general. Para ello, se enlistó la ayuda del equipo de tutores de escritura para la difusión del programa entre estudiantes, además de una serie de reuniones informativas con autoridades de las unidades académicas de la universidad y el envío de correos masivos a los potenciales usuarios. La plataforma fue positivamente recibida por académicos y funcionarios que reconocían en ella una oportunidad para hacerse cargo de una necesidad institucional. Sin embargo, y a pesar de la calidad de los materiales generados, la puesta en marcha estuvo afecta a muchas dificultades de uso y difusión que se discutirán a continuación.

En primer lugar, la plataforma Moodle requería que cada usuario se registrara con su número de identidad y algunos datos básicos (por ejemplo, carrera de origen y año de ingreso) para efectos de seguimiento. Sin embargo, en el proceso de registro se reportaron algunas dificultades, como el hecho de que los e-mails de confirmación eran, en muchos casos, detectados como spam. Así, la puesta en marcha inicial requirió una importante inversión de tiempo en soporte para los usuarios.

En segundo lugar, la plataforma tuvo un uso muy limitado durante su primer semestre. Se registraron 704 matriculados en los cursos ofrecidos, lo que resulta un número muy bajo comparado tanto con la audiencia principal (sobre 3.ooo estudiantes de primer año) como con la población total de usuarios potenciales (casi 30.000 estudiantes de pregrado y posgrado). Lo anterior se explica parcialmente por las dificultades de registro ya mencionadas, pero también evidencia la necesidad de una política estratégica de difusión mediante diversos canales y el conocimiento más profundo de la plataforma por parte de actores que pueden recomendar su uso (como profesores que soliciten evaluaciones escritas en sus cursos).

En tercer lugar, en el caso del laboratorio dirigido, la cantidad de estudiantes que lograron completar el curso de siete semanas fue muy bajo $(37,2 \%$ durante el primer semestre de implementación). Los alumnos que desertaron reportan que su carga académica regular no les permite dedicar el tiempo apropiado al desarrollo del curso de escritura y que, dado que este no ofrece creditaje, no existe un incentivo externo para completarlo. La interferencia de este agobio académico ocurrió a pesar de los esfuerzos del equipo de programar el curso en fechas en las que interviniera lo menos posible con períodos de evaluaciones o exámenes. No obstante, existe consenso tanto entre los estudiantes que completaron como aquellos que desertaron de que la secuencia didáctica, las estrategias trabajadas y el producto final esperado son de mucha utilidad para afianzar sus habilidades de escritura en contextos académicos.

Finalmente, la plataforma de LEA lab fue originalmente montada en servidores de un prestador externo y, a la hora de concretar los traspasos a los sistemas internos de la universidad, se detectó una incompatibilidad para manejar las bases de datos existentes. Lo anterior llevó a una completa reconsideración respecto a las posibilidades de man- 
tener la plataforma en el servidor externo, con los costos que ello implica, o su posible replicación en otro CMS compatible con los sistemas locales.

Por las dificultades reportadas, se tomaron algunas decisiones estratégicas para continuar el trabajo de la plataforma tras su primer semestre de uso:

Primero, se decidió recrear la plataforma en los servidores internos de la universidad, utilizando nuevamente la plataforma Moodle. En el traspaso se simplificó el proceso de registro del estudiante, al solicitar solo la inscripción con su número de identidad, y se escogió un diseño más sencillo que permitiera acceder rápidamente a la variedad de cursos ofrecidos. Además, se aprovechó esta instancia para desagregar los cuatro cursos en unidades más pequeñas, de modo de hacer más accesibles los contenidos: por ejemplo, se construyó un curso de Ortografía y se lo separó de los demás materiales de norma del español.

Segundo, se diseñó una nueva estrategia de difusión de la plataforma dirigida a estudiantes en proceso de escritura de sus trabajos de grado o de titulación, para así aprovechar la necesidad evidente de apoyo por parte de este grupo y el potencial de difusión informal entre sus contactos. Para ello se decidió generar una nueva serie de contenidos dirigidos explícitamente a la escritura de estos textos (normalmente, seminarios, tesis o memorias de grado o título), abordando sus partes típicas y algunas recomendaciones de metodología de la investigación, como la formulación de objetivos e hipótesis.

Tercero, se abandonó por ahora la iniciativa del laboratorio dirigido, pues se consideró que debe institucionalizarse su lugar para convertirlo en una acción efectiva de desarrollo de habilidades de escritura. El aseguramiento de condiciones mínimas para el normal desarrollo de un curso, entre las que predomina la disponibilidad y administración del tiempo, es esencial para el éxito de los cursos virtuales de escritura (Minter, 20I5). Por eso, se plantea negociar la asignación de creditaje al curso, para que pueda ser computado de manera oficial dentro de la carga académica del estudiante. Además, se requeriría asegurar algunas condiciones mínimas para los tutores que imparten el curso, entre ellas una remuneración justa, un número adecuado de alumnos, y una capacitación adecuada en aspectos de retroalimentación efectiva, didáctica de la escritura e instrucción en entornos virtuales (Mechenbier, 20I5).

Durante el año 2OI7 se ha explorado una nueva forma de utilizar la plataforma en cursos guiados, mediante una articulación con cátedras presenciales. En esta propuesta, la plataforma sirve para alojar actividades de apoyo para desarrollar la escritura con textos disciplinares, y el avance de cada estudiante se considera dentro de la evaluación final del curso presencial. Este método se probó en los cursos de "Investigación para las Ciencias Farmacéuticas", de la carrera de Química y Farmacia, y "Comunicación Académica en Ingeniería y Ciencias”, del Plan Común de Ingeniería. 


\section{Conclusiones a partir de la implementación}

Una plataforma como LEA lab viene a entregar una respuesta valiosa a la preocupación sobre la calidad de escritura de los estudiantes. Para el equipo, "nivelación” no se relaciona precisamente con el aseguramiento de que todos los estudiantes tengan las mismas habilidades de escritura; más bien, la nivelación se concibe como una oportunidad para poner a disposición de los estudiantes herramientas variadas que puedan incorporar para enfrentarse a los desafíos múltiples de cada uno de sus contextos. En este sentido, los materiales generados buscaban, ante todo, abarcar la mayor cantidad de estrategias transversales posibles. Punto aparte ha sido la generación de materiales contextualizados para generar géneros específicos en diferentes carreras, aprovechando los talentos de nuestro equipo de tutores pares (González-Álvarez, 2OI5b), los que han sido incorporados a la plataforma de autoaprendizaje desde 2016.

Ahora bien, para que la plataforma tenga el uso esperado debe existir en los estudiantes una percepción acerca de sus propias necesidades de instrucción. La Universidad de Chile tiene una cultura muy centrada en el éxito académico y es frecuente escuchar que solo "los mejores" estudiantes logran entrar a una de sus carreras; en ese contexto, reconocerse como mal escritor es una amenaza al prestigio de pertenecer a esta institución. Una plataforma virtual ofrece una ventaja en este sentido, porque permite que un estudiante preocupado por estos temas pueda consultarla de forma privada en su computador o celular. Sin embargo, para poder usar adecuadamente la plataforma, el estudiante necesita tener alguna idea sobre qué mejorar; así, es responsabilidad de todos quienes evalúan en el aula universitaria (docentes de cátedra, auxiliares y ayudantes) entregar una retroalimentación que permita detectar espacios concretos de mejora; no basta con decir a los estudiantes que deben escribir mejor o que tienen muchos errores de ortografía. Así, una plataforma de este tipo requiere también articularse con otros mecanismos que desarrollen estas discusiones más amplias; ese es el objetivo central del Programa LEA.

Por otro lado, y desde una perspectiva más general, la existencia de un espacio concreto para el desarrollo de la escritura, ya sea físico o virtual, se vuelve cada vez más obligatoria en universidades que se hacen cargo de las necesidades de alfabetización. Una oferta virtual tiene muchísimas ventajas, entre las que destaca la accesibilidad para los estudiantes desde cualquier espacio y dispositivo con acceso a internet, la posibilidad de consultar libremente contenidos relacionados con la tarea que estén realizando, y el establecimiento de algunos criterios de calidad de escritura que pueden convertirse en transversales para toda la comunidad universitaria. No obstante ello, existe una responsabilidad institucional en instalar una discusión sobre la importancia de la escritura y la necesidad de trabajarla desde el mismo inicio de los estudios universitarios. Es frecuente, en nuestra institución, que la escritura se considere como una habilidad menor durante todo el período previo a la producción de los trabajos de grado; la súbita aparición de altas exigencias en este respecto dificulta los procesos de graduación, las tasas de titulación oportunas y, sobre todo, afecta el autoconcepto de estudiantes que, habiendo com- 
pletado con éxito los desafíos en su formación hasta ese momento, se encuentran con una tarea muchas veces imposible de resolver. Así, es responsabilidad de académicos y administrativos prestar atención a estos asuntos desde el inicio de los estudios universitarios y guiar a aquellos estudiantes que se beneficiarían de acceder a recursos de apoyo en esta clase de plataformas.

Finalmente, la implementación de una plataforma como esta no puede ni debe entenderse como una forma de abaratar costos a la hora de hacerse cargo de esta necesidad de instrucción. El desarrollo de un laboratorio de escritura requiere inversión en el diseño, la escritura y la estructuración de los contenidos de cada curso, así como en el desarrollo de los objetos virtuales de aprendizaje, la implementación de la plataforma y el establecimiento de servidores y servicios de soporte informático. Estos últimos elementos, unidos a la atención constante para la actualización o creación de nuevos contenidos y la necesidad de establecer sistemas constantes de difusión, muestran la necesidad de contemplar una inversión permanente para el éxito de la iniciativa. 


\section{Referencias bibliográficas}

- Acevedo, I.; Cereceda, C.; Lovera, P.; Martínez, J.; Pereda, y Mery. (20I3, noviembre). Lectoescritura Académica y Cognición Situada. Ponencia presentada en el VII Congreso Internacional de la Cátedra Unesco de Lectura y Escritura. Córdoba, Argentina.

- Alzate-Medina, G. \& Peña-Borrero, L. (2OIO). La tutoría entre iguales: una modalidad para el desarrollo de la escritura en la educación superior. Universitas Psychologica, 9(I): 123-138. Obtenido el I2 de mayo de 2014 de http://www.redalyc.org/pdf/647/647I2I560IO.pdf

- Andrews, R.; Togerson, C.; Beverton, S.; Freeman, A.; Locke, T.; Low, G.; Robinson, A. \& Zhu, D. (2006). The effect of grammar teaching on writing development. British Educational Research Journal, 32(I), 39-55.

- Ávila, N.; González-Álvarez, P. \& Peñaloza, C. (20I3). Creación de un programa de escritura en una universidad chilena: estrategias para promover un cambio institucional. Revista Mexicana de Investigación Educativa, XVIII(57), 537-560. Obtenido el I2 de mayo de 2014 de http://www.redalyc.org/articulo.oa?id=I40257740I0030

- Ávila, N. \& González-Álvarez, P. (2OI4). On-line design as an opportunity to rethink writing pedagogies: a case study in the Pontificia Universidad Católica de Chile. Ponencia presentada en la 20I4 Conference on College Composition and Communication. Indianápolis, Indiana, EE.UU.

- Ávila, N. \& González-Álvarez, P. (20I6). Web 2.o y escritura universitaria: oportunidades para la escritura situada y el aprendizaje colaborativo en un curso online. En D. Riestra, S. M. Tapia \& M. V. Goicoechea (Eds.), Cuartas Jornadas Internacionales de Investigación y Prácticas en Didáctica de las lenguas y las literaturas (Tomo II) (pp. 798-8I7). Bariloche: Universidad Nacional de Río Negro.

- Badger, R. \& White, G. (2000). A process genre approach to teaching writing. ELT Journal, 54 (2), I53-I6o.

- Bhatia, V. (2002). A generic view of academic discourse. En J. Flowerdew (Ed.), Academic discourse (pp. 2I-39). Harlow, England: Longman.

- Carlino, P. (2003). Alfabetización académica: un cambio necesario, algunas alternativas posibles. Educere, 6(20), 409-420.

- Carlino, P. (20I3). Alfabetización académica diez años después. Revista Mexicana de Investigación Educativa, I8(57), 355-381. Obtenido el I2 de mayo de 2014 de http://www.redalyc.org/pdf/I4O/I4O25774OO3.pdf 
- Cassany, D. (1990). Enfoques didácticos para la enseñanza de la expresión escrita. Comunicación, Lenguaje y Educación, 6, 63-80.

- Concha, S. \& Miño, P. (2OI3, noviembre). Aprender a escribiry escribir para aprender en la universidad. Ponencia presentada en el XX Congreso de la Sociedad Chilena de Lingüística. Concepción, Chile.

- Didactext (2003). Modelo sociocognitivo, pragmalingüístico y didáctico parala producción de textos escritos. Didáctica (Lengua y Literatura), I5, 77-IO4. Obtenido el I2 de mayo de 2014 de http://revistas.ucm.es/index.php/DIDA/article/viewFile/DIDAo3o3IIoO77A/I9407

- González-Álvarez, P. (2OI4). Teorías implícitas sobre la escritura en docentes de Ingeniería Civil: articulación entre las creencias, la práctica y el lugar de la producción escrita en la carrera. Tesis para optar al grado de Magíster en Lingüística Aplicada. Pontificia Universidad Católica de Valparaíso, Chile.

- González-Álvarez, P. (2OI5a). El ‘curso basal’ para la enseñanza de la escritura en la universidad: una propuesta de enseñanza que concilia la formación general con la orientación disciplinar. En C. Muse (Ed.), Cátedra UNESCO Lectura y Escritura: comunidades, rupturas y reconstrucciones, vol. 3 (Lectura y escritura en el nivel superior) (pp.I38-I45). Córdoba: Universidad Nacional de Córdoba.

- González-Álvarez, P. (20I5b, septiembre). Didáctica de los géneros de formación: generación de materiales didácticos mediante investigación-acción con tutores pares. Póster presentado en el VIII Simpósio Internacional de Estudos de Gêneros Textuais. São Paulo, Brasil.

- González-Álvarez, P. \& Izquierdo, C. (20I4, abril). Un enfoque de género y proceso para la producción de textos en el aula universitaria. Ponencia presentada en el Tercer Congreso de Pedagogía de la Cátedra Unesco de Lectura y Escritura. Osorno, Chile.

- Greenhow, C.; Robelia, B., \& Hughes, J. E. (2009). Learning, Teaching, and Scholarship in a Digital Age: Web 2.o and Classroom Research: What Path Should We Take Now? Educational Researcher, 38(4), 246-259. doi:Iо.3IO2/оог3г89Xо933667I

- Hillard, V. \& Harris, J. (2003). Making writing visible at Duke University. Peer Review, Fall 20O3: I5-I7.

- Kim, Y. \& Kim, J. (2005). Teaching Korean University writing class: balancing the process and the genre approach. Asian EFL Journal, 7(2) [en línea]. Obtenido el 2I de junio de 2016 de http://www.asian-efl-journal.com/June_O5_yk\&jk.pdf. 
- Martinez, D. \& Olsen, L. (20I5). Online Writing Labs. En B. L. Hewett \& K. E. DePew (Eds.), Foundational Practices of Online Writing Instruction (pp. I83-2Io). Fort Collins, Colorado: The WAC Clearinghouse y Parlor Press. Obtenido el 2I de junio de 2016 de http:// wac.colostate.edu/books/owi/

- Martínez, J. A. (2OII). Revisión y evaluación: por el mejoramiento de la escritura académica universitaria. Actualidades Pedagógicas, 57, I4I-I67. Obtenido el 2I de junio de 2016 de https://revistas.lasalle.edu.co/index.php/ap/article/view/554

- Mechenbier, M. (20I5). Contingent Faculty and OWI. En B. L. Hewett \& K. E. DePew (Eds.), Foundational Practices of Online Writing Instruction (pp. 227-249). Fort Collins, Colorado: The WAC Clearinghouse y Parlor Press. Obtenido el 2I de junio de 20 or6 de http:// wac.colostate.edu/books/owi/

- Mick, C. S. \& Middlebrook, G. (2015). Asynchronous and Synchronous Modalities. En B. L. Hewett \& K. E. DePew (Eds.), Foundational Practices of Online Writing Instruction (pp. I29-I48). Fort Collins, Colorado: The WAC Clearinghouse y Parlor Press. Obtenido el 2i de junio de $20 I 6$ de http://wac.colostate.edu/books/owi/

- Minter, D. (20I5). Administrative Decisions for OWI. En B. L. Hewett \& K. E. DePew (Eds.), Foundational Practices of Online Writing Instruction (pp. 2II-225). Fort Collins, Colorado: The WAC Clearinghouse y Parlor Press. Obtenido el 2I de junio de 2016 de http:// wac.colostate.edu/books/owi/

- Nordin, S. \& Mohammad, N. (2006). The best of two approaches: process/genre based approach to teaching writing. The English Teacher, XXXV, $75^{-8} 5$.

- Parodi, G. (I999). Relaciones entre lectura y escritura. Valparaíso: Ediciones Universitarias de Valparaíso.

- Peñaloza, C. (20I3, noviembre). Un plan de formación de habilidades comunicativas en el terapeuta fonoaudiólogo: desde la alfabetización lingüistica hacia la inmersión profesional. Ponencia presentada en el XX Congreso de la Sociedad Chilena de Lingüística. Concepción: Chile.

- Sánchez, E.; González, A. \& García, R. (2002). Competencia retórica. Una propuesta para interpretar las dificultades de comprensión. Psicothema, I4(I), 77-85.

- Stoller, F. (20O2). Project work: a means to promote language and content. En J. Richards \& W. Renandya (Eds.), Methodology in Language Teaching, an anthology of current practice (pp. Iо7-IIg). Nueva York: Cambridge University Press.

- Swales, J. M., (1990). Genre Analysis: English in academic and research settings, Cambridge: Cambridge University Press. 
-Warnock, S. (20I5). Teaching the OWI course. En B. L. Hewett \& K. E. DePew (Eds.), Foundational Practices of Online Writing Instruction (pp. I5-I8I). Fort Collins, Colorado: The WAC Clearinghouse y Parlor Press. Obtenido el 2I de junio de 2016 de http://wac. colostate.edu/books/owi/

-Warschauer, M. (2007). The paradoxical future of digital learning. Learning Inquiry, I(I), 4I-49. doi:IO.IOO7/sII5I9-OO7-OOOI-5

- Williams, J., Cureton, R., Moses, C. \& Vavra, E. (I985). Four comments on "Grammar, grammars, and the teaching of grammar". College English, 47 (6), 64I-649. 